\title{
Management of Acute Appendicitis During the COVID-19 Pandemic is Significantly Different: A Retrospective Single UK Hospital Study
}

\author{
Mohammad Noah Hasan \\ Khan (1D) \\ Abdul Basit Jamal $\mathbb{D}^{2}$ \\ Ahmad Faraz ${ }^{3}$ \\ Hassan Shafique $^{4}$ \\ Muhammad Umar Rasool ${ }^{5}$ \\ Muhammad Waqas llyas ${ }^{6}$ \\ Muhammd Hamza Jamshed (iD) \\ Waleed Riaz $^{8}$ \\ Mehwish Usman ${ }^{9}$ \\ 'Trauma and Orthopedic Surgery, Royal \\ Victoria Hospital, Belfast, Northern \\ Ireland; ${ }^{2}$ Trauma and Orthopedic \\ Surgery, Ittefaq Hospital Trust, Lahore, \\ Pakistan; ${ }^{3}$ Department of General \\ Surgery, Royal Victoria Hospital, Belfast, \\ Northern Ireland; ${ }^{4}$ Trauma and \\ Orthopedic Surgery, Royal London \\ Hospital, London, UK; ${ }^{5}$ Trauma and \\ Orthopedic Surgery, North Manchester \\ General Hospital, Manchester, UK; \\ ${ }^{6}$ Trauma and Orthopaedics University \\ Hospital Southampton, Southampton \\ Hampshire, UK; ${ }^{7}$ Trauma and \\ Orthopedics, The Hillingdon Hospitals \\ NHS Trust, Uxbridge, UK; ${ }^{8}$ General \\ Surgery Royal Victoria Hospital, Belfast, \\ Northern Ireland; ${ }^{9}$ Trauma and \\ Orthopedics, Manchester Royal \\ Infirmary, Manchester, UK
}

Correspondence: Mohammad Noah

Hasan Khan

Trauma and Orthopedic Surgery, Royal

Victoria Hospital, Belfast, Northern

Ireland

Email noah_khan@hotmail.com
Introduction: The COVID-19 pandemic has led to a change in the delivery of acute and emergency surgical services. With emphasis on reducing unnecessary operative intervention and performing more CT scans, there has been a shift in managing acute appendicitis conservatively. We evaluate the impact of this shift on the management of acute appendicitis. Methods: A single UK centre retrospective study evaluating patients with suspected acute appendicitis pre-COVID-19 rota (18 March 2020) and post-COVID rota implementation. Data including demographics, inflammatory markers, imaging, mode of management and operative findings were collected. Logistic regression with SPSS was used to determine which factors were associated with conservative management and treated with antibiotics.

Results: A total of 161 patients were analysed, 82 pre-COVID19 and 79 post-COVID19. Of the pre-COVID-19 patients, $67.07 \%$ underwent appendicectomy while the rest were conservatively managed; $24.3 \%$ of these patients underwent a CT scan only. Post-COVID-19, $22.78 \%$ of patients underwent appendicectomy with a higher percentage of diagnostic CT scans performed, $43 / 79(54.4 \%, \mathrm{p}<0.001)$. The proportion of histologically normal appendicectomies was significantly reduced in the post-COVID-19 era $(12.78 \%$ vs $0.00 \%$; p-value 0.001). Logistic regression analysis showed a normal WCC to be associated with greater likelihood of conservative management. No conservatively managed patients returned to theatre in the 30-day follow-up period.

Conclusion: Due to the restrictions imposed by the post-COVID-19 rota, a greater proportion of patients were managed conservatively with comparable patient outcomes. The approach also led to fewer negative appendicectomies owing to greater reliance on imaging. Keywords: appendicitis, COVID-19, imaging, non-operative management

\section{Introduction}

The COVID19 pandemic was declared by the World Health Organization on 11 March 2020. ${ }^{1}$ Due to the expected influx of patients with COVID19 related respiratory symptoms and pressure on hospital services including intensive care, several surgical guidelines including those released by the Royal College of Surgeons advised a change in the delivery of surgical services. ${ }^{2,3}$ This has led to restrictions in emergency and elective services with focus on delivery of urgent cancer care, emergency presentations and deferment of non-urgent cases. ${ }^{3}$ Furthermore, laparoscopic surgery was restricted due to the potential risk of aerosol generation which limited operations in the emergency setting with strict regulation on personal protective equipment. ${ }^{2,3}$ This has led to a re-evaluation of managing surgical conditions in the emergency setting. 
Acute appendicitis is amongst the most common general surgical emergencies with a $7 \%$ lifetime risk. ${ }^{4}$ The standard treatment of acute appendicitis remains laparoscopic appendicectomy. ${ }^{5}$ Preoperative CT scan rate in the UK is significantly lower compared with other regional high-income countries. ${ }^{17}$ Traditional UK surgical practice has avoided use of CT scanning to reduce radiation exposure. This has however led to a higher negative appendicectomy rate. ${ }^{6-8}$ Several studies have evaluated the possibility of managing acute appendicitis with antibiotics alone and shown this to be safe and effective..$^{5-14}$ Furthermore, at the outset of the COVID19 pandemic, there was great anxiety amongst surgeons with the risk from aerosol generation from laparoscopic surgery, consequently there was a shift towards open appendicectomy which is inherently associated with increased morbidity. ${ }^{15}$

The aim of this study was to analyse the change in management of patients presenting with suspected acute appendicitis as an emergency during the CoVID19 pandemic and its impact on patient outcomes. The key metric being the ongoing safe delivery of this emergency surgical service. We further examined factors that would affect surgical decision making between conservative and operative management. ${ }^{7,16}$

\section{Materials and Methods}

All patients ( $>14$ years) presenting with acute right iliac fossa pain were analysed over a 6-month period between December 2019 and May 2020. A cut-off date of 18 March 2020 was used to divide the patient cohort into pre-COVID19 (Pre) and post-COVID19 (Post) categories as this was the date for alteration in the local surgical protocols to deal with the COVID19 pandemic as well as the date for application of the Royal College of Surgeons' guidance.

Patients suspected of acute appendicitis presenting to the emergency general surgery admissions were included. Those under 18 years or with previous appendicectomies were excluded. The data were gathered prospectively at a single UK district general hospital. Data including patient demographics, inflammatory markers, imaging, surgical approach, length of stay and re-admission were documented. We further followed patients to determine whether operative intervention was required within 30 days following discharge in those cases managed conservatively who had confirmed appendicitis.

All CT scans were of the abdomen and pelvis, with intravenous contrast (Omnipaque 300) administered. All the CT scans were reviewed by consultant radiologists. The surgical approach was either standard open technique performed with "Grid-iron" or "Lanz" incision in the right iliac fossa or 3-port laparoscopic technique. Those undergoing conversion to open surgery were also included. In cases of conservative management of confirmed cases of acute appendicitis, patients were managed with antibiotics as per local trust guidelines.

All statistical analysis was performed using SPSS version 25. A logistic regression model to evaluate factors predictive of operative intervention used the binary outcome of operation versus conservative management for discrete and continuous variables. A comparison between pre-COVID19 rota and post-COVID19 rota management of appendicitis was undertaken. The results were analysed using Mann-Whitney $U$-test and $T$-test.

\section{Results}

Overall, 161 patients were analysed during the entire study period including 89 females and 72 males. Of these patients 82 underwent assessment and management for acute appendicitis prior to 18 March 2020 when the COVID19 rota was imposed whereas 79 patients underwent assessment and management after this date.

Of the Pre cohort, the median age was 30 years (range: 14-87) while in the Post cohort it was 39 (17-88) years (p-value 0.001). There were significantly more male patients in the Pre cohort, with $43(52.4 \%)$ male and 39 (47.6\%) female patients compared with 29 (36.7\%) males and $50(63.3 \%)$ females (p-value 0.045). The median duration of symptoms in both of the groups was reported the same at 1 day (1-14 days)(Table 1$)$.

\section{Investigations}

The inflammatory marker results are summarised in Table 2. In the Pre-COVID19 group, the patients had higher CRP and white cell counts which are statistically significant, and 31 (37.8\%) patients underwent an ultrasound scan compared with $29(36.7 \%)$ in the PostCOVID19 cohort (Table 3). Among the patients who had an ultrasound scan performed, the appendix was not

Table I Patient Characteristic Having Appendicitis

\begin{tabular}{|l|l|l|l|}
\hline Parameter & Pre (n=82) & Post (n=79) & p-value \\
\hline Age & $30(14-87)$ & $39(17-88)$ & $0.00 I$ \\
Gender (male) & $43(52.4 \%)$ & $29(36.7 \%)$ & 0.045 \\
Duration of symptoms & $\mathrm{I}(\mathrm{I}-14)$ & $\mathrm{I}(\mathrm{I}-14)$ & 0.96 \\
\hline
\end{tabular}


Table 2 Inflammatory Markers in Patients Presenting with Suspected Acute Appendicitis

\begin{tabular}{|c|c|c|c|c|c|}
\hline Inflammatory Markers & & Frequency Pre (Percentage) & Frequency Post (Percentage) & Median & p-value \\
\hline WCC & $\begin{array}{l}4-10^{* 9} / \mathrm{L} \\
11-15^{* 9} / \mathrm{L} \\
>15^{* 9} / \mathrm{L}\end{array}$ & $\begin{array}{l}24(29.3 \%) \\
27(32.9 \%) \\
31 \quad(37.8 \%)\end{array}$ & $\begin{array}{l}39(49.4 \%) \\
23(29.1 \%) \\
17(21.5 \%)\end{array}$ & $13.5: 13.0$ & 0.004 \\
\hline CRP & $\begin{array}{l}<5 \mathrm{mg} / \mathrm{dl} \\
5-50 \mathrm{mg} / \mathrm{dl} \\
51-100 \mathrm{mg} / \mathrm{dl} \\
>100 \mathrm{mg} / \mathrm{dl}\end{array}$ & $\begin{array}{l}14(17.1 \%) \\
17(20.7 \%) \\
19(23.2 \%) \\
32(39.0 \%)\end{array}$ & $\begin{array}{l}28(35.4 \%) \\
20(25.3 \%) \\
13(16.5 \%) \\
18(22.8 \%)\end{array}$ & $67.5: 33.5$ & 0.002 \\
\hline
\end{tabular}

Table 3 Radiological Investigations Performed in Patients Presenting with Suspected Acute Appendicitis

\begin{tabular}{|l|l|l|l|}
\hline Parameter & Pre (n=82) & Post (n=79) & p-value \\
\hline US Scan & $31(37.8 \%)$ & $29(36.7 \%)$ & 0.90 \\
CT Scan & $20(24.3 \%)$ & $43(54.4 \%)$ & 0.000047 \\
MRI Scan & 0 & $\mathrm{I}$ & \\
\hline
\end{tabular}

visualized in $25(86 \%)$ in the Pre group compared with 15 (48.4\%) in the Post cohort. US scan was reported as "normal" in 4 of the Pre cohort patients compared with 3 in the Post cohort. Acute appendicitis was diagnosed on US scan in 13 Pre cohort patients while none of the Post cohort had it diagnosed with this radiological modality.

Twenty (24.4\%) patients underwent CT scans as part of the diagnostic workup in the Pre group versus 43 (54.4\%) patients in the Post group $(\mathrm{p}<0.001)$. Furthermore, amongst those who had a CT scan, $7(35 \%)$ vs $23(54.8 \%)$ had a normal appendix with the remaining having appendicitis (Table 4). In 13 (65\%) of Pre group patients CT scan showed appendicitis compared with 20 (45.2\%) in the Post group. No patient with a normal reported CT scan was operated upon. No patient in this study was found to have generalized peritonitis as a complication of acute appendicitis. The intraoperative findings in both groups showed a significant decrease in the frequency of intra-operatively normal-

Table 4 Incidence of Findings from CT Abdomen-Pelvis with IV Contrast in Patients Presenting with Suspected Acute Appendicitis

\begin{tabular}{|l|l|l|}
\hline CT Findings & $\begin{array}{l}\text { Pre Frequency } \\
\text { (n=20) }\end{array}$ & $\begin{array}{l}\text { Post Frequency } \\
\text { (N=43) }\end{array}$ \\
\hline Normal appendix & $7(35.0 \%)$ & $23(54.8 \%)$ \\
Uncomplicated appendicitis & $7(35.0 \%)$ & $14(31.0 \%)$ \\
Localized abscess & $5(25.0 \%)$ & $2(4.8 \%)$ \\
Pelvic abscess & $0(0 \%)$ & $0(0)$ \\
Perforated appendix & $\mathrm{I}(5.0 \%)$ & $4(9.5 \%)$ \\
Generalized peritonitis & $0(0)$ & $0(0)$ \\
\hline
\end{tabular}

looking appendices in the post-COVID19 group, 7 (12.7\%) vs $0(0 \%)$ (Table 5$)$.

In addition, within the pre-COVID19 group, 31 (56.4\%) patients underwent appendicectomy without any form of imaging i.e. US or CT scan. However, in the postCOVID19 group, none of the 18 patients underwent appendicectomy on clinical evaluation alone, with all patients having either a CT scan, US scan or both preoperatively.

\section{Mode of Management and Predictive Factors}

The majority of patients in the Pre group underwent operative management for suspected acute appendicitis (55; $67.07 \%)$ compared with $18(22.78 \%)(\mathrm{p}=0.013)$ in the Post group. A statistically significant proportion of patients in the Post group were managed non-operatively (61; $77.21 \%$ ) compared with the Pre group (27; 32.92\%). Almost all of the operations in this study were performed laparoscopically (Table 6).

Logistic regression analysis identified that a raised level of CRP was predictive of requirement for operative intervention $(\mathrm{p}<0.05)$ in the Pre group (Table 7). A normal white cell count (WCC) was predictive for nonoperative management in the Post group (Table 8).

Table 5 Intraoperative Findings of Patients Operated on for Acute Appendicitis

\begin{tabular}{|l|l|l|l|}
\hline Intraoperative Findings & $\begin{array}{l}\text { Frequency } \\
\text { (Percentage) } \\
\text { n= 55 }\end{array}$ & $\begin{array}{l}\text { Frequency } \\
\text { (Percentage) } \\
\text { n= I8 }\end{array}$ & p-value \\
\hline Normal appendix & $7(12.7 \%)$ & $0(0 \%)$ & 0.001 \\
Uncomplicated appendicitis & $34(61.8 \%)$ & $11(61.1 \%)$ & \\
Localized abscess & $9(16.3 \%)$ & $2(11.1 \%)$ & \\
Pelvic abscess & $1(1.8 \%)$ & $(5.6 \%)$ & $4(22.2 \%)$ \\
Perforated appendix & $4(7.3 \%)$ & $0(0 \%)$ & \\
Generalized peritonitis & $0(0 \%)$ & \\
\hline
\end{tabular}


Table 6 Mode of Management of Patient with Acute Appendicitis

\begin{tabular}{|l|l|l|l|}
\hline Mode & Pre $\mathbf{n = 8 2}$ & Post $\mathbf{n = 7 9}$ & p \\
\hline Operated & $55(67.07 \%)$ & $18(22.78 \%)$ & 0.013 \\
Non-operated & $27(32.92 \%)$ & $61(77.21 \%)$ & \\
Laparoscopic & $52(94.54 \%)$ & $18(100 \%)$ & \\
\hline
\end{tabular}

Table 7 Logistic Regression Analysis to Identify Factors Predictive of Operative Intervention for Acute Appendicitis in Pre-COVIDI9 Cohort of Patients Presenting to the Emergency Department

\begin{tabular}{|l|l|l|}
\hline Variable & OR + (95\% Cl) & P-value \\
\hline Age & $0.955(0.887-1.029)$ & 0.228 \\
Gender & $0.384(0.051-2.905)$ & 0.354 \\
Duration of symptoms & $0.506(0.237-1.078)$ & 0.315 \\
WCC & $0.801(0.672-42.292)$ & 0.113 \\
CRP & $15.430(1.278-18.247)$ & 0.031 \\
CT scan & $0.230(0.20-2.660)$ & 0.239 \\
US scan & $0.379(0.044-3.268)$ & 0.377 \\
\hline
\end{tabular}

Table 8 Logistic Regression Analysis of Factors Predictive Conservative Management in Patients Presenting with Acute Appendicitis Post Covid

\begin{tabular}{|l|l|l|}
\hline Variable & Odds Ratio (95\% CI) & p-value \\
\hline Age & $1.002(0.962-1.043)$ & 0.930 \\
Gender & $1.359(0.303-6.104)$ & 0.689 \\
Duration of symptoms & $0.757(0.440-1.303)$ & 0.315 \\
WCC & $0.143(0.024-0.836)$ & 0.031 \\
CRP & $0.190(0.025-1.445)$ & 0.109 \\
CT scan & $2.329(0.326-16.650)$ & 0.400 \\
US scan & $0.586(0.076-4.542)$ & 0.609 \\
\hline
\end{tabular}

\section{Complications and Length of Stay}

Median delay to surgery was 1 day (range $0-2$ days) across the groups (Table 9). The median length of stay in the hospital was 1 day (range 1-7 days) across both groups (Table 9). Six of the operated patients developed superficial wound infections with 1 developing a deep abscess. One patient developed a chest infection and 1 required re-operation for stump leak. Five patients from the Pre group and 2 patients from the Post group were re-admitted following appendicectomy with miscellaneous complaints which were managed conservatively. This was not statistically significant. No conservatively managed patient of confirmed appendicitis required operative intervention in the follow-up period.

\section{Discussion}

Acute appendicitis is one of the most common presentations to the emergency general surgical "take-in". ${ }^{4}$ This
Table 9 Complications and Length of Stay

\begin{tabular}{|l|l|l|}
\hline Parameter & Pre $\mathbf{n = 5 5}$ & Post $(\mathbf{n}=\mathbf{1 8})$ \\
\hline Delay to surgery & I day (0-2) & 2 days $(I-5)$ \\
Length of stay & $\mathrm{I}(1-7)$ & $\mathrm{I}(\mathrm{I}-7)$ \\
Complications & $14(25.45 \%)$ & $3(16.66 \%)$ \\
\hline
\end{tabular}

Abbreviations: $\mathrm{P}$ value, probability of obtaining results to test hypothesis; $\mathrm{N}$, number of patients; $95 \% \mathrm{Cl}$, confidence interval kept at $95 \%$.

study provides one of the first reports on the impact of COVID19 on the management of this condition in a district general hospital. Our study suggests the implementation of restrictions on the surgical service due to COVID19 did not affect the safe delivery of this emergency surgical service. Furthermore, there was no worsening of patient outcomes for those undergoing appendicectomy with no differences in readmission or complication rates. This study has clearly shown a greater reliance on imaging in the form of CT scans and use of conservative management for patients with suspected acute appendicitis. Although clinical workup is dependent on the individual patient, our study highlights that with increasing use of cross-sectional imaging, the rate of negative appendicectomy has significantly reduced. Furthermore, patients managed conservatively with antibiotics did not show increased evidence of adverse events and did not require surgery within our follow-up period. The diagnosis of appendicitis is traditionally established clinically in current UK standard practice. ${ }^{16}$ No formal scoring system has been universally adopted, although we found that a normal WCC was predictive of patients more likely to undergo conservative management.

The use of CT scanning for diagnosis of acute appendicitis is traditionally low in the UK which has led to higher negative appendicectomy rates compared with the rest of Europe. ${ }^{7}$ This was further shown in the RIFT audit $^{16}$ where normal appendicectomy rates of $28.2 \%$ in females and $12.1 \%$ in males were reported. Moreover, this was related to lower use of radiological imaging to diagnose appendicitis in the UK compared with Europe. The majority of CT scans in post-COVID19 patients were reported as normal which facilitated conservative management and reduced the overall number of operative interventions. With the increased suspected risk from COVID19 in the operative setting, particularly in laparoscopic procedures, combined with the greater morbidity of open surgery, prior imaging has become necessary practice to reduce operative exposure both for the patient and 
surgeon. Although there is utility of imaging prior to operation in these patients to avoid unnecessary operations, many with uncomplicated appendicitis also underwent operative intervention, reflecting the lack of a standardised approach to management of this condition in the UK. Factors influencing this include individual consultant preference and patient choice.

Finally, there are limitations to this study. It is from a single centre where the majority of patients with confirmed appendicitis, had an uncomplicated form. This would make the condition more amenable for conservative management of acute appendicitis. In addition, we do not have the long-term follow-up data to determine which patients managed conservatively would ultimately develop recurrent appendicitis requiring operative intervention. The recently published CODA trial has established a $30 \%$ rate of appendicectomy in conservatively managed patients within 90 days despite showing non-inferiority of conservative management. Nonetheless, this study is an important contribution to demonstrate the impact of COVID19 on management of appendicitis and the service adjustments that have been required. One trend that was seen in our study was the use of CT scans in the post COVID19 group. This may have been because at the time of assessing the abdomen it would give the option of scanning the chest as well, and as at the outset of the pandemic we did not have reliable methods to diagnose the virus, a CT could detect lung changes. This might have influenced the enthusiasm to proceed to CT, however we do not have enough evidence to come to this conclusion.

\section{Conclusions}

Management of acute appendicitis in the post-COVID19 era was significantly adapted leading to alterations in service delivery. Patient outcomes remained comparable. Due to the restrictions, there was significantly greater use of cross-sectional imaging and conservative management of confirmed cases reflecting forthcoming changes in UK practice. Long-term follow-up data is required.

\section{Ethical Statement}

This research project was registered as a quality improvement study with the clinical governance department in our hospital, after being assigned a QI number we were given permission to review and compare data, as this was for obtaining retrospective data with no human subjects or prospective requirement, authors were exempted from getting an ethical review board certification.

\section{Funding}

No funding was needed for this study.

\section{Disclosure}

The authors report no conflict of interest for this study. Revised manuscript has complied with relevant data protection and privacy regulations.

\section{References}

1. Cucinotta D, Vanelli M. WHO declares COVID-19 a pandemic. Acta Bio Med. 2020;91(1):157-160.

2. Brucher B, Nigri G, Tinelli A, et al. COVID-19: pandemic surgery guidance. 4 Open. 2020;3(19):1.

3. Tuechab A, Gangloffc F, Di Fiorebc P, et al. Strategy for the practice of digestive and oncological surgery during the Covid-19 epidemic. Elsevier. 2020;157(3):7-12.

4. Hajibandeh S, Hajibandeh S, Hobbs N, Mansour M. Neutrophil-tolymphocyte ratio predicts acute appendicitis and distinguishes between complicated and uncomplicated appendicitis: a systematic review and meta-analysis. Am J Surg. 2020;219(1):154-163. Epub 2019 Apr 27. PMID: 31056211. doi:10.1016/j.amjsurg.2019.04.018

5. Podda M, Gerardi C, Cillara N. Antibiotic treatment and appendectomy for uncomplicated acute appendicitis in adults and children. Ann Surg. 2019;270(6):1028-1040. doi:10.1097/SLA.0000000000003225

6. Rogers W, Hoffman J, Noori N. Harms of CT scanning prior to surgery for suspected appendicitis. Evid Based Med. 2015;20(1):3-4. Epub 2014 Nov 27. PMID: 25429870. doi:10.1136/ebmed-2014-110075

7. van Rossem CC, Bolmers MD, Schreinemacher $\mathrm{MH}$, et al. Diagnosing acute appendicitis: surgery or imaging? Colorectal Dis. 2016;18(12):1129-1132. doi:10.1111/codi.13470

8. Andersson M, Kolodziej B, Andersson RE; STRAPPSCORE Study Group. Randomized clinical trial of appendicitis inflammatory response score-based management of patients with suspected appendicitis. Br J Surg. 2017;104(11):1451-1461. doi:10.1002/bjs. 10637

9. Hall N, Eaton S, Stanton M, et al. Active observation versus interval appendicectomy after successful non-operative treatment of an appendix mass in children (CHINA Study): an open-label, randomised controlled trial. Pubmed. 2017;2(4):253-260. doi:10.1016/ S2468-1253(16)30243-6

10. Abongwa HK, Cervellin G, Tarasconi A, et al. Antibiotics alone for uncomplicated acute appendicitis in high operative risk adult patients: analytical review of RCTs and proposal of evidence based treatment decision. Acta Biomed. 2016;87(3):334-346. PMID: 28112705.

11. Hansson J, Körner U, Khorram M. Randomized clinical trial of antibiotic therapy versus appendicectomy as primary treatment of acute appendicitis in unselected patients. Pubmed. 2009;96 (5):473-481. doi:10.1002/bjs. 6482

12. Styrud J, Eriksson S, Nilsson I, et al. Appendectomy versus antibiotic treatment in acute appendicitis. a prospective multicenter randomized controlled trial. World J Surg. 2006;30(6):1033-1037. doi:10.1007/ s00268-005-0304-6

13. Ozgüç H. Appendectomy versus antibiotic treatment in acute appendicitis: a prospective multicenter randomized controlled trial. World J Surg. 2007;31(3):615;author reply 616. doi:10.1007/s00268-006-0521-7

14. Flum DR, Davidson GH, Monsell S, et al. A randomized trial comparing antibiotics with appendectomy for appendicitis. $N$ Engl J Med. 2020;383(20):1907-1919. doi:10.1056/NEJMoa2014320 
15. Ielpo B, Podda M, Pellino G, et al. Global attitudes in the management of acute appendicitis during COVID-19 pandemic: ACIE Appy Study. BJS. 2020.

16. Nepogodiev D, Wilkin RJ. Appendicitis risk prediction models in children presenting with right iliac fossa pain (RIFT Study): a Prospective, Multicentre Validation Study. Lancet. 2020;4(4):271-280.
17. Chan J, Fan S, Mak K. Pre-operative imaging can reduce negative appendectomy rate in acute appendicitis. Ulster Med J. 2020;89 (1):25-28.

\section{Publish your work in this journal}

The Journal of Multidisciplinary Healthcare is an international, peerreviewed open-access journal that aims to represent and publish research in healthcare areas delivered by practitioners of different disciplines. This includes studies and reviews conducted by multidisciplinary teams as well as research which evaluates the results or conduct of such teams or healthcare processes in general. The journal covers a very wide range of areas and welcomes submissions from practitioners at all levels, from all over the world. The manuscript management system is completely online and includes a very quick and fair peer-review system. Visit http://www.dovepress.com/testimonials. php to read real quotes from published authors. 